\title{
An IEEE 802.11 protocol test-bed in industrial environments using personal computing devices
}

\author{
J. Vales-Alonso*, F. J. Pérez-Martínez, A. S. Martínez-Sala, \\ E. Egea-López, F. Ponce-Marín, J. García-Haro \\ Departament of Information Technologies and Communications, \\ Polytechnic University of Cartagena, Spain. \\ * Corresponding author. Address: Campus Muralla del Mar, 30202, Cartagena, Spain. \\ phone: +34 968326 588, fax: +34 968325 973, e-mail: javier.vales@upct.es
}

\begin{abstract}
IEEE 802.11 is being currently considered to develop telecommunications infrastructures in industrial environments. However, in such environments there may exist a high level of Electromagnetic Fields (EMF) contamination, due to the machinery in use. Wireless LANs are sensitive to this type of hazard, and therefore the performance of the protocols may be affected inside the industrial facilities. In this paper we conduct a study of the wireless link performance in these industrial scenarios, using personal computing devices. Such equipments will be likely used by employees rather than conventional computers. Since the results do not characterize the environment itself, but the set of environment and hardware, a proper measurement test-bed must include direct measurements with these devices. Besides, due to limitations in these equipments, a specific protocol to measure pairs of packet error probability and latency is also developed. Results reveal a significant linear dependency between both magnitudes, and a large correlation coefficient in the measurements under high EMF activity at mid-large ranges (distances greater than 5 meters).
\end{abstract}

Index Terms-EMF, IEEE 802.11, Industrial environments

\section{INTRODUCTION}

Wireless networking has driven significant advances in information technologies during the last decade. For instance, the Global Mobile System (GSM), the Universal Mobile Telecommunication System (UMTS) and the Wireless Local Area Networks (WLAN) have achieved a high penetration in the market, both in domestic and office use. Currently, the IEEE 802.11 protocol, and its versions $\mathbf{a}, \mathbf{b}$ and $\mathbf{g}$, stand out among the WLAN proposals [1]. IEEE 802.11 allows for a faster and more economic network deployment than conventional wired LANs. Indeed, in industrial enviroments, where it is sometimes quite difficult or even impossible to setup a wired installation, wireless one allows the development of handy facilities. However, in this type of environments there may exist a high level of Electromagnetic Fields (EMF) contamination, due, among other effects, to the machinery in use. WLANs are sensitive to this kind of hazard, and therefore the performance of the protocols may be affected inside the industrial facilities. Thus, a systematic study of the wireless link performance in these scenarios is mandatory to establish the limits in the protocol operation.

Additionally, the devices that installed in these facilities are usually different from those employed for common use: employees demand mobility, a requirement that is met by means of Personal Computing Devices (PCD), e.g. Personal Digital Assistant (PDA) or Tablet PCs. Besides, in next years new applications based on the Wireless Sensor Network (WSN) paradigm will allow distributed data collection and actuation on specific points. Since the radio transceivers and hardware of both types of devices (PCDs and WSNs) have different characteristics and properties (e.g. antenna design, processing speed, memory) than conventional ones, measurements are not independent from the hardware used to obtain them. That is, results do not characterize the environment itself, but the set environment-hardware. Thus, a proper measurement test-bed must include direct measures with these specific devices. In addition, most studies only take into consideration Packet Error Probability (PEP) or bit error rate. However, a characterization for a specific environment and hardware should also include measurements of the end-to-end delay, that is, the latency. Both magnitudes, the PEP and the latency characterizes the communication system from the MAC (Medium Access Control) layer perspective. Nevertheless, both magnitudes are not, a priori, independent. Therefore, the measurement software must sample pairs of this random bivariate variable (PEP, latency) to characterize the environment.

In this paper we have measured the influence of EMF in industrial noisy environments for a wireless IEEE 802.11b link. In several experiments we have computed the packet error probability and the latency. Similar studies have been already conducted in other works (see [2]), however authors use conventional wireless devices (laptops with WI-FI Network Interface Cards, NICs) and only measure the PEP. In our testbed, we use commercial PDAs to take into account the variations introduced by these equipments, and we want to measure the latency and the PEP for each sample. This requirement forces us to develop a specific measurement protocol, since we have not direct access to the statistics and network facilities at the MAC layer in these PDAs.

The goal of such test-bed is twofold. First, we aim to identify if there are clear trends that distinguish the industrial environments from the office ones. In this case, we want to quantify them and provide parameters that measure the different degrees of industrial activity. Second, based on the data collected we will formulate and empirical-based stochastic model for the industrial environments, that depends on the formely identified parameters. Additionally, this probabilistic 
description of the channel can be used to implement realistic channel models for simulation with low computational cost [3]. In this work we focus on the first point, leaving the latter point as future work.

The rest of this paper is organized as follows. Section II contains the related work and a concise description of the IEEE 802.11 b protocol. Section III describes the protocol developed to collect statistics. Section IV shows the results obtained in the measurement trials, and points out their most significant properties. Finally, section V concludes and describes possible future works.

\section{RELATED WORK}

Wireless communication has been used for long in industry, however its use has been limited to solve very specific problems, and has rarely been used as the main networking means. Currently, emerging wireless technologies, like the IEEE 802.11, have an increasing potential to solve general problems and to serve as the basis of new telecommunications framework for the industry. The key of wireless success in the industrial market will be to match the industrial requirements of security, safety and robustness (see [4]). Such issues require a sound channel characterization and the adaptation of high-level protocols. Probably, the IEEE 802.11 is the most promising solution in this field. IEEE 802.11 is based on the CSMA/CA (Carrier Sense Multiple Access/Collision Avoidance) protocol [1]. That is, it uses the collision avoidance RTS/CTS handsake before actual packet transmission, to avoid the hidden node issue. In addition, packet reception is directly acknowledged at the MAC layer, due to the error-prone media. If an ACK is not received, the protocol uses the Automatic Repeat reQuest (ARQ) mechanism to recover from this error The number of the ARQ retransmissions can be configured in the wireless interface. The $802.11 \mathrm{~b}$ version of the standard establishes the $2.4 \mathrm{GHz}$ Industrial Scientific and Medical (ISM) Band as the operation band.

A number of works have analyzed the performance of IEEE $802.11 \mathrm{~b}$ under different working scenarios. For instance, in [5] the authors experimentally study the effect of sharing the ISM band of $802.11 \mathrm{~b}$ with other devices (Bluetooth). They conclude that the throughput is considerably affected if both systems share the same coverage area. In [2] authors measure the bit error rate directly at the MAC layer in an industrial environment using a special wireless NIC compliant with the IEEE 802.11 standard. They remark the high time variability in the experiments, and conclude that although results are not representative in all industrial environments, there are common effects: large error bursts mixed with other error free bursts, but with a tendency to bursty errors. The project [6] is a great-effort test-bed intended to collect a comprehensive set of data to research on the behavior of operational wireless networks. Finally, a probabilistic description of wireless links would be very useful to derive models for simulation. In [3], real measurements of WSN link properties are used to to compute network connectivity graphs for simulation. These graphs reduce computational cost while providing a very accurate description of real channels.

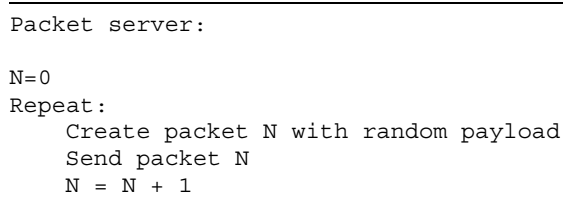

Measurement client:

For each measurement point

Receive packet I

Errors $=0$

Packet $\mathbf{s}=0$

Samples $=0$

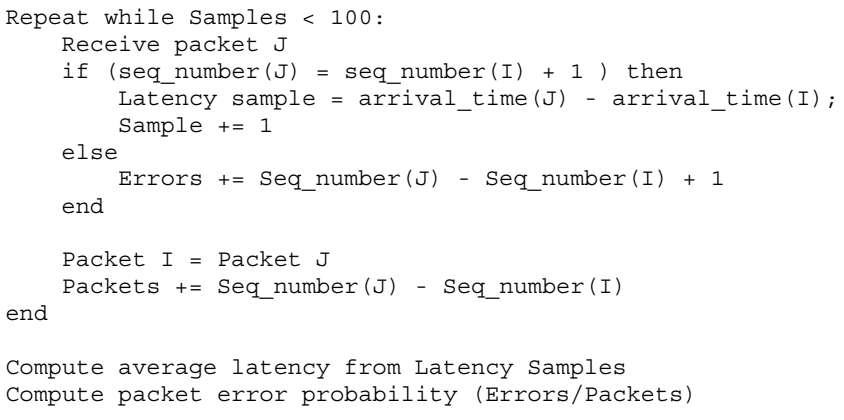

TABLE I

MEASUREMENT PROTOCOL

\section{MEASUREMENT PROCEDURE}

\section{A. Measurement protocol}

The measurements were performed using commercial PDAs HP iPAQ Pocket PC h5500. These devices do not allow to use a direct programming interface with the MAC layer, nor the collection of statistics at the MAC interface. This fact forces us to develop a specific protocol to measure the packet error probability and the latency, as seen by the IP layer. This protocol was developed directly on top of the UDP/IP stack. The pseudo-code description of the proposed protocol is illustrated in Table I. The measurement algorithm consists of two processes, a packet server, that continuously sends packets, and a measurement client, that captures pairs of packet error probability and latency values. For each measurement point the client averages 100 samples of latency, and computes the associated PEP as the number of erroneus packets divided by the total number of received packets. Notice that latency samples are only computed if two consecutive packets are received. Therefore, the total numer of received packets can be larger than 100. Additionally, as can be seen in Section IV this measurement protocol discretizes the sampling space in the PEP axis. The accuracy is 1/\#Packets, since the number of packets is greater or equal than 100 , the accuracy is in the order of 0.01 .

The protocol is implemented in $\mathrm{C}$, and cross compiled for strongARM using a conventional Intel P-IV PC. The code was run on the iPAQ using the Linux Familiar ${ }^{1}$ 0.8.2 distribution, running Linux kernel version 2.4.18.

\footnotetext{
${ }^{1}$ http://familiar.handhelds.org/
} 


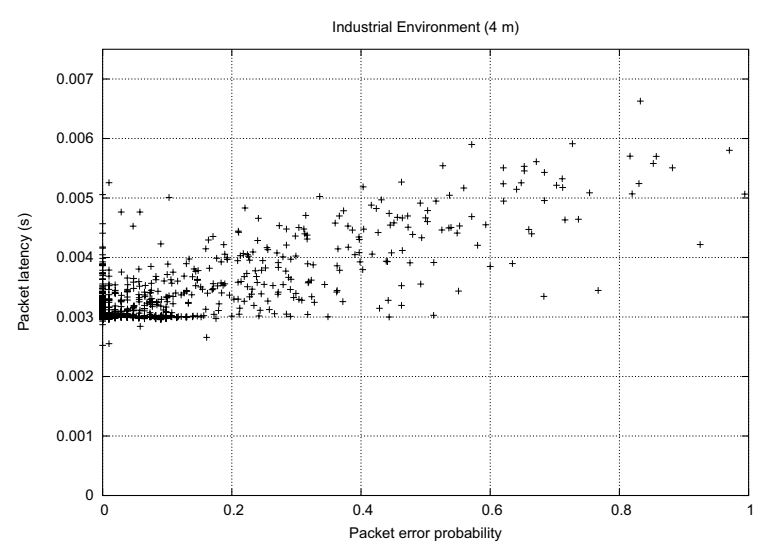

Fig. 1. Example of raw data

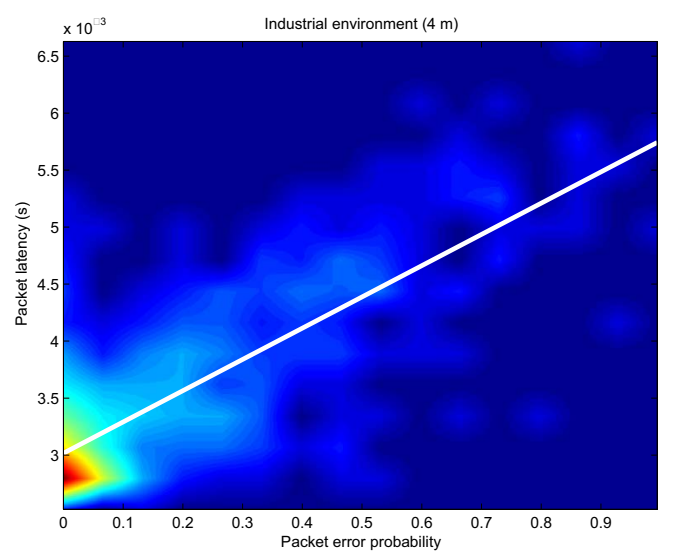

Fig. 2. Example of post-processed data

\section{B. Post-processing}

After each measurement experiment, a set of pairs (PEP, latency) is obtained. This set has been post-processed to ease the interpretation of the results. For instance, Fig. 1 shows a planar representation of all the points measured (latency is expressed in seconds) in one experiment in the industrial environment described in the next section. This representation is not clear if many points are concentrated in a small area. To avoid this issue we have computed a 2D histogram based on the measured points, and linearly interpolated them. These histograms are then represented as colormaps where red colors denote a high probability density, and blue ones a lower probability density. Therefore, we obtain a graphical representation of the probability density. For instance, Fig. 2 corresponds to the data set of Fig. 1. In addition, the line of regression of Latency on PEP (Latency $=m P E P+b)$ for the measured set of points is also computed and shown in the corresponding figure. The correlation coefficient, $\rho$, is also calculed for each data set.

\section{RESUlts}

In this section the experimental results obtained are presented and discussed. Table II shows the main configuration parameters used for all the experiments. In each test two

\begin{tabular}{l|l} 
Parameter & Value \\
\hline Transmission Rate & Auto \\
Payload & 1492 bytes \\
Frequency & $2.437 \mathrm{GHz}$ (Channel 6) \\
ACK retries & 4
\end{tabular}

TABLE II

WIRELESS NIC CONFIGURATION

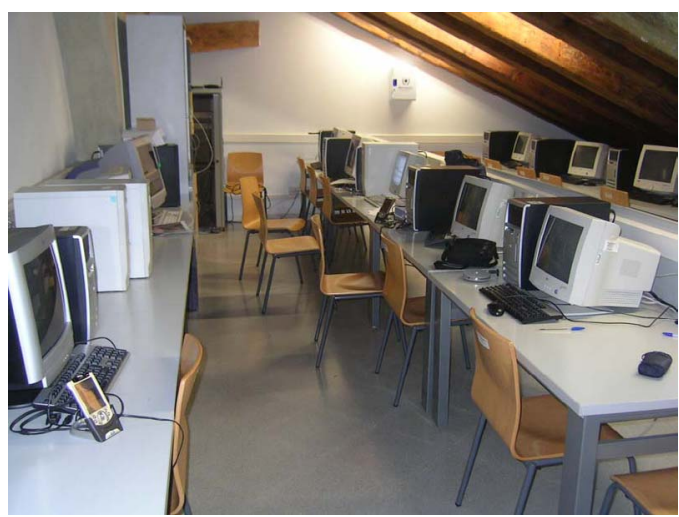

Fig. 3. IT-6 Laboratory, Polytechnic University of Cartagena, Spain

stations were situated at a static distance $d$. In this position 4,000 points were captured (about 400,000 samples) ${ }^{2}$. The tests were performed in two scenarios:

1) IT-6 laboratory at the Polytechnic University of Cartagena, Spain (see Fig. 3). It is composed of several workstations. This environment is similar to common office configurations. In this scenario, we performed several tests to adjust the measurement protocol. These tests are also used to compare with the results from the industrial environment. For instance, Fig. 5 depicts the results obtained in this environment for a distance $d=$ $19 \mathrm{~m}$. The results indicate a high and puntual probability concentration around the mean value of the pair PEP, latency; e.g. $(0.0022,0.0030 \mathrm{~s})$ in the previous example. Figure 5 also shows a zoom of the $[0,0.02] x[2.8$, $3.4]$ area. In this zoom the effect of discretization in the PEP axis -due to the measurement protocol- is noticeable. The slope of the line of regression is 0.0008 . Additionally, the measured correlation coefficient was less than 0.5 in all the experiments performed, and equal to $\rho=0.1508$ for the scenario of Fig. 5 .

2) Industrial facilities of the Estructuras del Mar Menor factory, in Cartagena, Spain (see Fig. 4). This is a typical factory floor, with several moving employees performing soldiering and working with heavy machinery. Fig. 6 shows the probability density representations measured for the distances $d=0,4,8,16,32$ and 48 meters. The slopes of the lines of regression are $m=0.0018$, $0.0030,0.0030,0.0032,0.0031$ and 0.0029 , respectively. The correlation coefficients measured are $\rho=0.3982$, $0.8336,0.9588,0.9634,0.9532$ and 0.8448 , respectively.

${ }^{2}$ In all the experiments, except for the industrial configurations at $d=32 \mathrm{~m}$ and $d=48 \mathrm{~m}$, where only 2,000 points were captured due to safety reasons. 


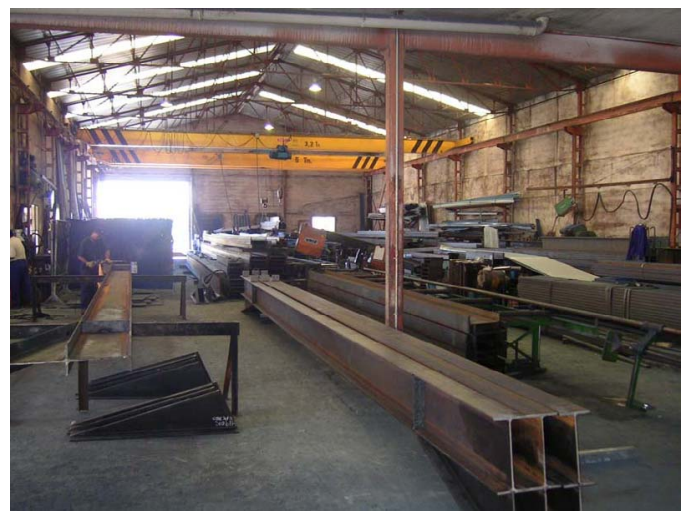

Fig. 4. Industrial facilities of Estructuras Mar Menor, Spain

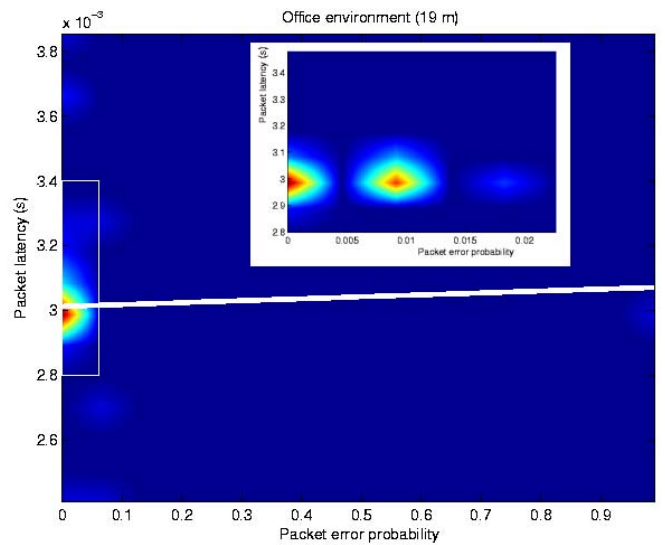

Fig. 5. IT-6 Laboratory, $19 \mathrm{~m}$ test

The results exhibit a clear linear dependency of both magnitudes, PEP and latency, for distances $d \geq 8 \mathrm{~m}$. On the contrary, for the trials carried out at a less distance, the shapes of the probability density function are similar to the punctual distributions obtained for the office environment. Besides, in the trial at $d=16$ $\mathrm{m}$ there was a soldiering activity peak, this effect has been captured in the corresponding probability density function, as can be observed in Fig. 6. The probability is more spread along the line of regression. The correlation coefficient was, indeed, maximum in this trial.

In addition, the ordered PEP samples are depicted in Fig. 7 for $d=4,8,16$ and 32 meters. A high burstiness can be observed in these figures. That is, periods of low error rates are mixed with periods of high error rate. This property is also present in the rest of the tests.

\section{A. Discussion}

Based on the previous results we can conclude that,

1) There is a very significant change between industrial and office environment, for distances in the mid-large range $(d>5 \mathrm{~m})$. In the industrial environment the correlation coefficient is larger $(\rho \geq 0.8)$ than in office environments $(\rho \leq 0.5)$. Besides, the slope of the line of regression also suffers a notable change, in the office and low range industrial environments it is significantly lower than in the mid-large range industrial tests. Indeed, it stabilizes around the 0.0030 value in these scenarios.

2) As the distance increases, the probability density is distributed along the line of regression of Latency on PEP. Therefore, there is a strong linear dependency of PEP and latency in the industrial environments.

3) Peaks of EMF activity cause higher correlation coefficients $(\rho \approx 0.95)$, and a noticeable probability density spread.

4) As in [2] a high burtstiness was observed in the experiments. Such high error rate periods seem to be directly related with the industrial activity peaks (e.g. soldiering).

\section{CONCLUSIONS AND FUTURE WORK}

In this paper we develop a measurement tool to compute the probability density function associated to the bivariate random variable (Packet Error Probability, Latency). Using this tool we perform several tests in office and industrial environments. The results show that there is a clear dependency among both random variables in the industrial environment, and that the dispersion of the probability is small if the transmitter and the receiver are closer. If distance between them is larger or there is EMF activity, the dispersion is greater. These results have been computed using personal computing devices, rather than conventional computers, to take into account the effect of the hardware.

In our future work we aim to develop a simulator based in the previous measurements, considering also the stochastic nature of the system. This simulator will enable us to compute the reliability and performance of applications in an industrial facility, without the need for direct test (at least in the first stages of the application development). In addition, we will extend the measurements obtained performing new tests with different hardware (e.g. Mica2 motes commonly employed in WSN) and with different configuration parameters, to study how they affect the results.

\section{ACKNOWLEDGEMENTS}

This work has been cofunded by the Economy, Industry and Innovation Council, with the SOLIDMOVIL project (2I04SU044), supported by Fundacion Seneca, from the Region of Murcia with the ARENA Project (00546/PI/04), and by the Spanish Research Council with the ARPaq project (TEC2004-05622-C04-02/TCM) and the CSI-RHET project (TEC2005-08068-C04-01/TCM).

\section{REFERENCES}

[1] ANSI/IEEE Std. 802.11, "Part 11: Wireless LAN Medium Access Control (MAC) and Physical Layer (PHY) Specifications", 1999

[2] A. Willing, M. Kubisch, C. Hoene y A. Wolisz, "Measurements of a Wireless Link in an Industrial Environment Using an IEEE 802.11Compliant Physical Layer". IEEE Trans. of Industrial Electronics, vol. 49, no. 6, 2002.

[3] A. Cerpa, J. L. Wong, L. Kuang, M. Potkonjak, D. Estrin, "Statistical Model of Lossy Links in Wireless Sensor Networks", Proc. IEEE/ACM Int. Conf. on Information Processing in Sensor Networks, Los Angeles, CA, 2005. 

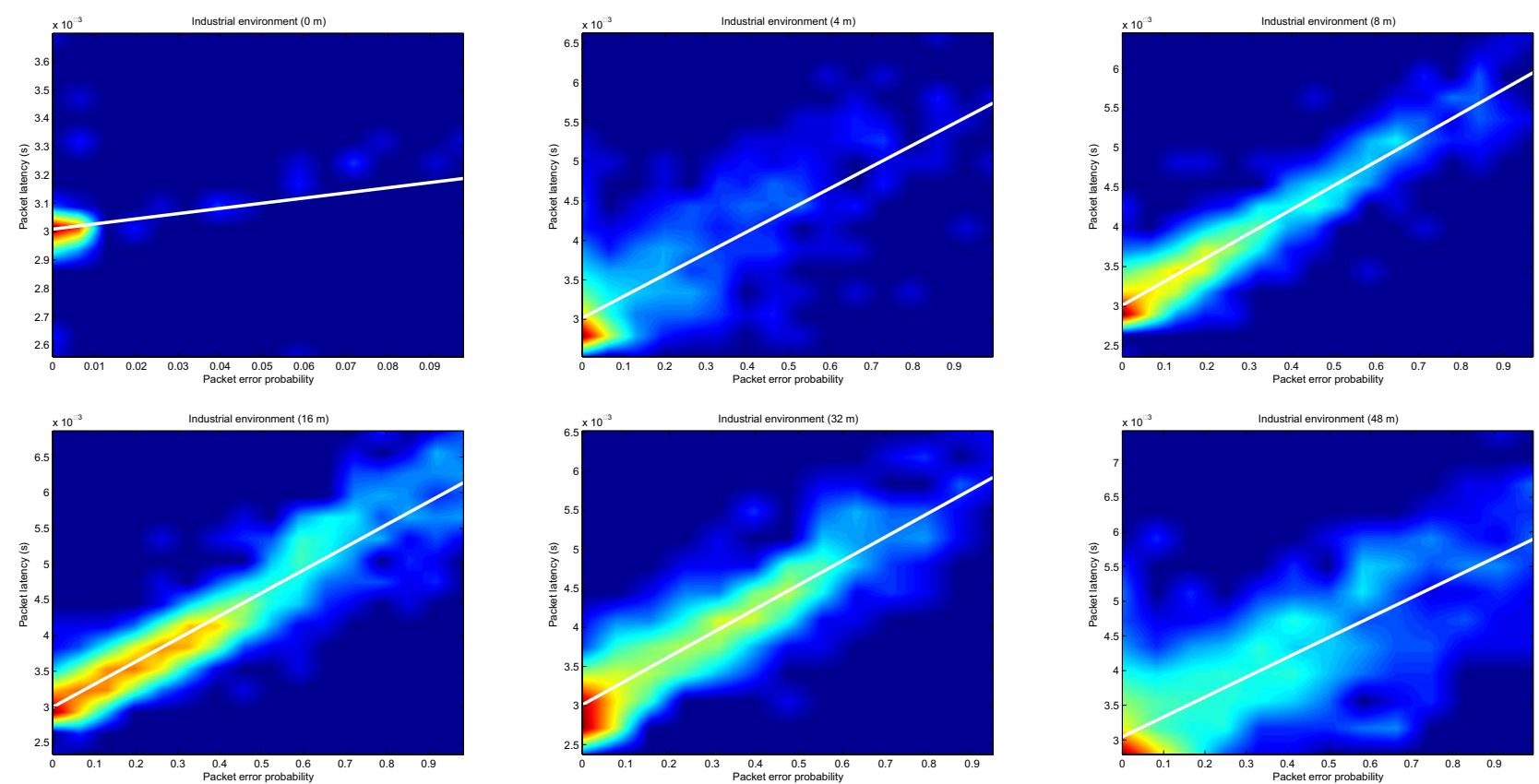

Fig. 6. Probability density function colormaps for the industrial enviroment
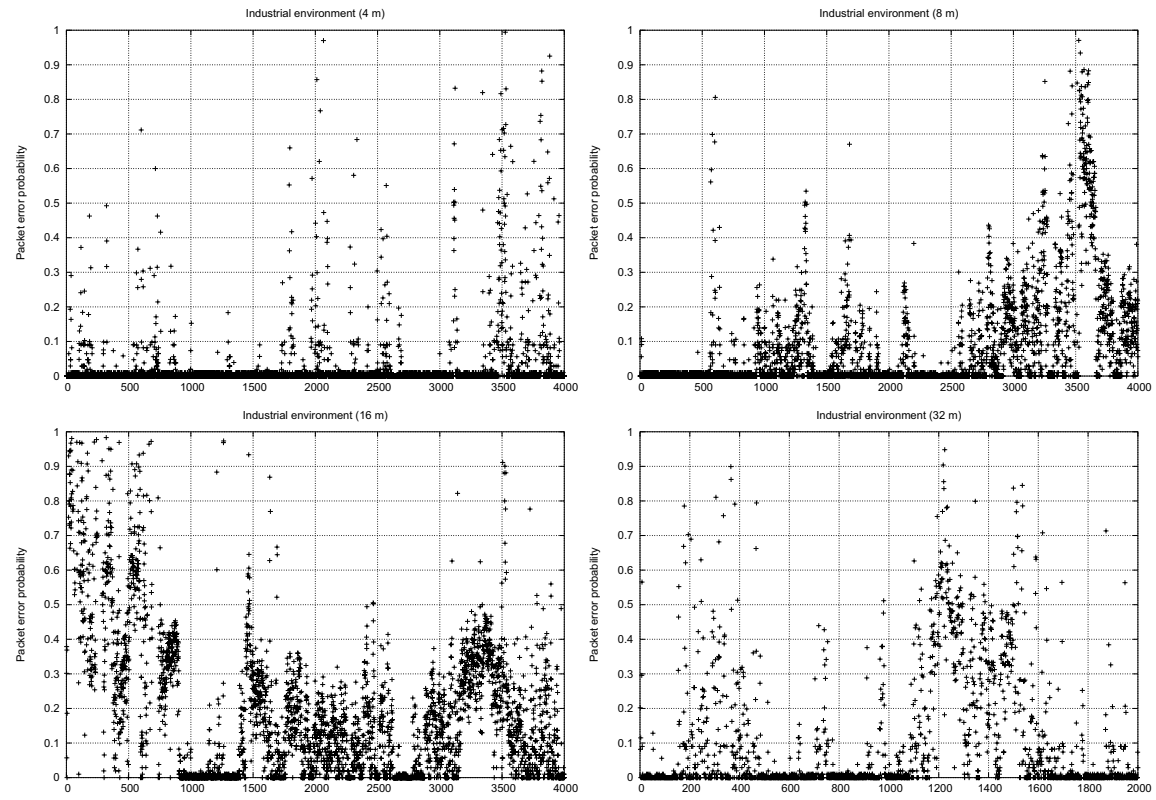

Fig. 7. PEP temporal samples for the industrial environment

[4] E. Egea-Lopez, A. Martinez-Sala, J. Vales-Alonso, J. Garcia-Haro, J. Malgosa-Sanahuja, "Wireless Communications Deployment in Industry: A Review of Issues, Options and Technologies", Elsevier Computers in Industry, 56(1): 29-53, 2005.

[5] J. del Prado Pavón y S. Choi, "Experimental Study on Co-existence of 802.11b with Alien Devices". Wireless Communication and Networking, Philips Research USA, 2001.

[6] http://uluru.ee.unsw.edu.au/ tim/measure.11 IEEE 802.11 Measurements Project. Tim Moors, Robert Malaney. Mahbub Hassan. 